\title{
TEGHNIQUES OF MEASUREMENT OF IMPACT RESPONSE OF THE THORAX IN BIOMECHANICAL EXPERIMENTS- PART II: METHODS OF ANALYSIS
}

\author{
by G.S. Nusholtz
}

\begin{abstract}
Experimental impact testing of human surrogates provides basic biomechanical information for understanding the kinematic response of the human body. Procedures for instrumenting these human surrogates with electromechanical transducers are described in the article entitled "Techniques of Measurement of Impact Response of the Thorax in Biomechanical Experiments-Part I: Instrumentation." (Part $l$ appears in November $1986 E / T$.) The following is the methods of analysis.
\end{abstract}

\section{TRANSFER FUNCTIONG}

For blunt thoracic impacts to a human surrogate, a transformation of simultaneously monitored transducer timehistories can be used to obtain the frequency-response functions of transducer time-histories of remote points. In the case of any two transducer timehistories, such as impact force and acceleration of a given point on the thorax, a transformation of the form

$$
(X)(\omega)=F[F(t) / F[A(t)]
$$

can be calculated from the transformed quantities, where $\omega$ is the given frequency and $F[F(t)]$ and $F[A(t)]$ are the Fourier transforms of the impact force and acceleration time-history, respectively. Transfer functions ${ }^{1}$ are complex. valued functions, which, for the purpose of emphasis, can be described by their magnitudes and phase angles.

G.S. Nusholtz is Assistant Research Scientist, The University of Michigan, Ann Arbor, MI.

\section{THANBFER FUNCTION BPECTRAL COHERENCE}

The coherence function $\mathrm{Cxy}^{2}(\omega)$ is not, strictly speaking, a transfer function, but a measure of the quality of a given transfer function at a given frequency.

$\operatorname{Cxy}^{2}(\omega)=|\operatorname{Gxy}(\omega)|^{2} /[\operatorname{Gxx}(\omega) \operatorname{Gyy}(\omega)]$

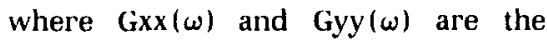
power-spectral densities of the two signals, respectively. Power-Spectral Density is a Fourier transform of each signal's autocorrelation. $|\mathrm{Gxy}(\omega)|^{2}$ is the Cross-Spectral Density function squared. Cross-Spectral Density is the Fourier transform of the cross-correlation of the two signals and $\omega$ at the given frequency. By definition, $0 \leq=$ $\operatorname{Cxy}^{2}(\omega) \leq=1$. Values of $\operatorname{Cxy}^{2}(\omega)$ near 1 indicate that the two signals may be considered causally connected at that frequency. Values significantly below 1 at a given frequency indicate that the transfer function at that frequency cannot accurately be determined. In the case of an input-output relationship, values of $\operatorname{Cxy}^{2}(\omega)$ less than 1 indicate that the output is not attributable to the input and is perhaps due to extraneous noise. The coherence function in the frequency domain is analogous to the correlation coefficient in the time domain. The coherence function can be used to determine the useful range of the data in the frequency domain. When attempting to understand the response of the thorax in the frequency domain, it is necessary to remove as much noise as possible. One method is through a "non-linear shift variant" filter developed at The University of
Michigan Transportation Research Institute (UMTRI).

\section{TIME DOMAIN AND FILTER OPERATION}

There are two aspects associated with the implementation of this filter: (1) characterizing the noise, and (2) estimating segments of the time-history using a power series.

In the case of an impact with a freemoving mass into the thoracic biological system, the input and output response of that system can be viewed as causal, provided that it has not been filtered with a noncausal filter. (In our practice, this is done by not filtering beyond the anti-aliasing filtering done before digitization.) A period of time, before impact (time zero), is recorded and digitized. For this period of time, it is assumed that fluctuation in the signal is a result of random or Gaussian noise with no aspect related to the response of interest. Further, it is assumed that the mean of these values is the true estimate of the value during this interval. The mean can then be subtracted from the rest of the signal to remove certain aspects of very low-frequency noise from the entire time-history. The variance associated with this mean can then be used to help in removing higher fre quency noise.

The principal property of the Taylor series is that if a function [C(t)], such as force or acceleration, is analytical in the vicinity of a given point in time, then a power series, in which the coefficients are related to the derivatives of the function being approximated, can be constructed. 


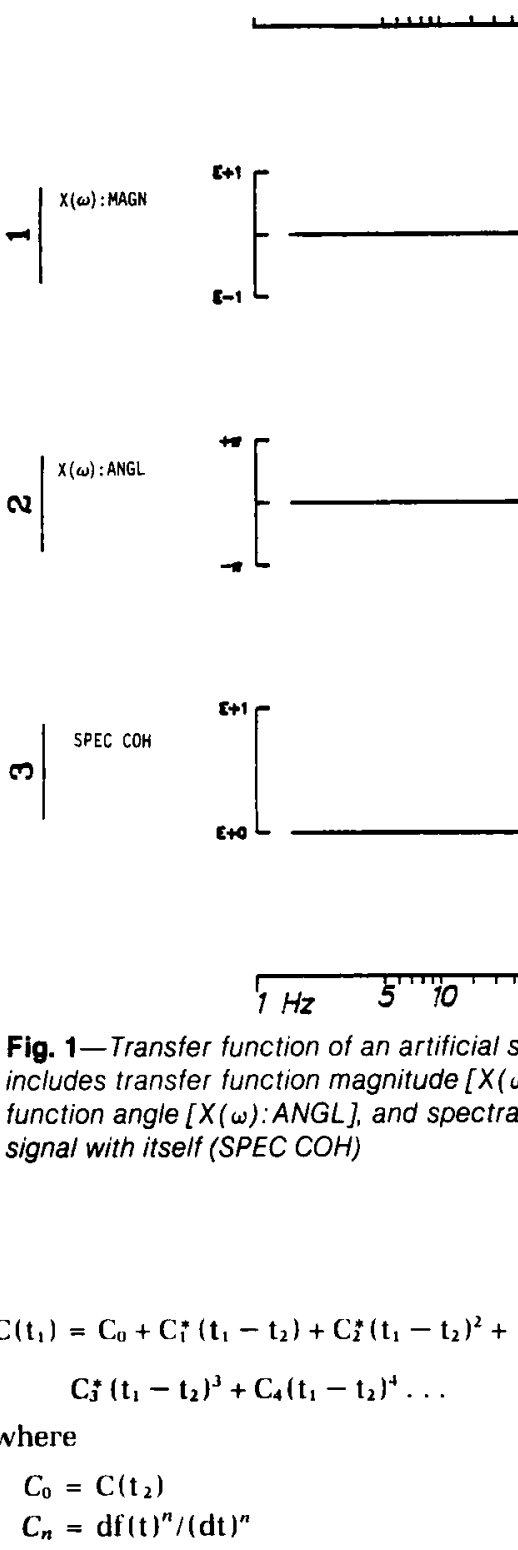

If the assumption is made that the noise observed in the interval of the signal before time zero is similar to that seen after time zero, then for any such analytical signal, a power series at each point can be constructed to estimate its neighborhood. (In the case of a digital time-history of a thoracic impact, this means no pathological changes exist in values between points.) However, digital time-histories present several problems that do not occur with truly analytical time-histories. Some of these are: (1) the neighborhood of any given point is quantized and cannot always be made as small as needed, (2) a true derivative cannot be taken at a point and each given approximation of the derivative of any order must include local points, (3) the higher order derivatives must include at least as many points as there are orders, and (4) for rapidly varying signals, the accuracy of the computer may limit the ability to approximate the time-history through power series.
The UMTRI filter employs a power series to estimate the value of any given point on a thoracic time-history using a least-squares approach. The algorithm works in the following man. ner: each "half" neighborhood of a given point is evaluated in terms of a series of power series. If the first two points before the given point, plus the given point, can be estimated in the least-squares sense with first-order equations so that the variance is less than or equal to the variance obtained from before time zero, then it is assumed that the least-squares approximation represents a truer estimate of the signal than the original data and the reconstruction is stored.

Next, four points are evaluated (three from the "half" neighborhood plus the given point) with a first-order fit. If the reconstruction proves to be a better estimate, using the criteria stated above of the true signal, then it is also stored. When an increase in points no longer allows the signal to be approximated with a linear fit, a second order fit is employed, and so on until sixth order is reached (sixth order was chosen on the basis of round-off error). If either sixth order is reached, or a given number of points is reached the number of points is chosen based upon a compromise between execution time and accuracy), the process is started over again on the next point. If any area cannot be fitted with a power series using a least-squares fit, it is left alone and the next set of points is chosen. After the entire thoracic time-history has been evaluated, each point has either been reconstructed a number of times or left alone. (The number of reconstructions depends on the neighborhood of the given point.) Each point then becomes the sum of its reconstruction divided by the number of reconstructions.

This UMTRI filter is similar in nature to a moving-average filter, except that the coefficients are determined by the values of the points being filtered as well as by the number of points. In this regard, the filter is shift-variant. In addition, if two signals are added, the noise before time zero changes and the filter will have a different effect on the time-history of the summed signals, than it would have on filtering the signals first and then summing them. Thus, the filter is non-linear.

This filter has been used repeatedly at UMTRI on over 10,000 different 

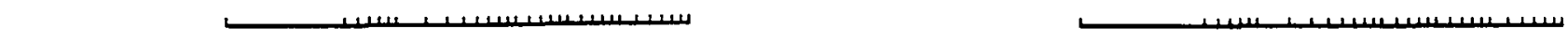
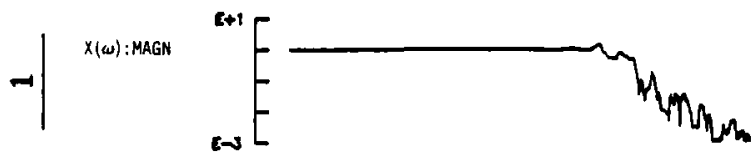

$\Omega \mid \times(\omega):$ ANGL

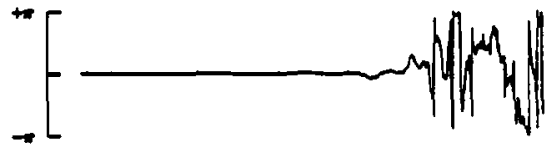

SPEC $\mathrm{COH}$

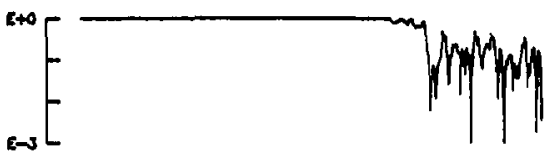

$1 \mathrm{~Hz} 5$ "10 50 '100 $500 \mathrm{~Hz}$

Fig. 3-Transfer function for the base signal with noise 1 (variable 2 in Fig. 2). Figure includes transfer function magnitude $[X(\omega): M A G N]$, transfer function angle $[X(\omega): A N G L]$, and spectral coherence for the two signals (SPEC COH)
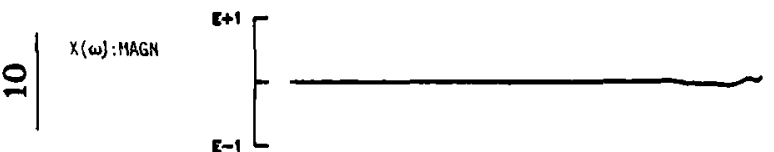

$=\quad x(\omega):$ :ANGL<smiles>CCCCC</smiles>

Q

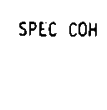

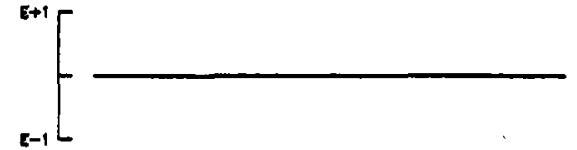

ऽ

$X(\omega)$ : ANGL
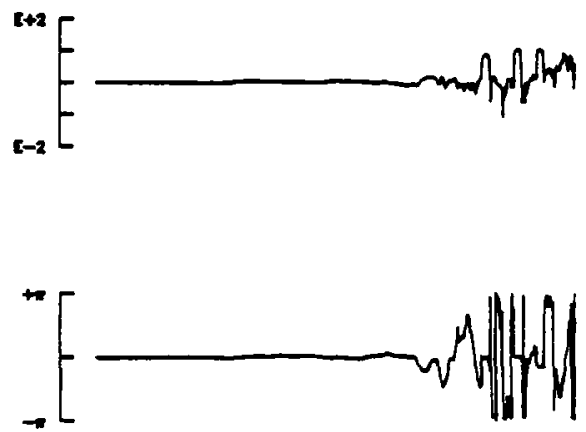

5) SPEC COH

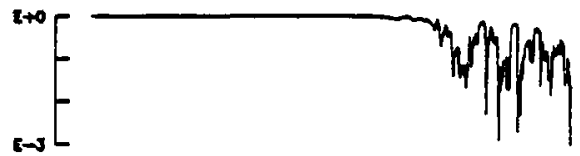

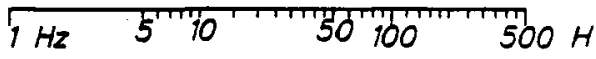

Fig. 4-Transfer function for base signal with filtered base signal with noise 1. Figure includes transfer-function magnitude [X( $\omega): M A G N]$, transfer-function angle $[X(\omega): A N G L]$, and spectral conerence for the two signals (SPEC COH)

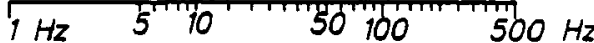

Fig. 5-Transfer function for base signal (variable 1 in Fig. 2) with filtered base signal with noise 1 . However, the signal-to-noise ratio is reduced to 2.0 percent from 33 percent. Figure includes transfer-function magnitude $[X(\omega): M A G N]$, transfer-function angle $[X(\omega): A N G L]$, and spectral coherence for the signal with itself (SPEC COH)

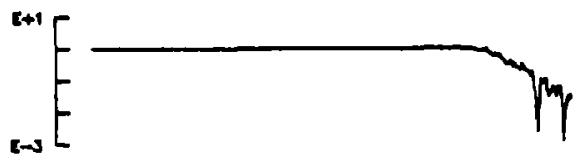

$\mathbf{v}$ $X(\omega)$ :ANGL

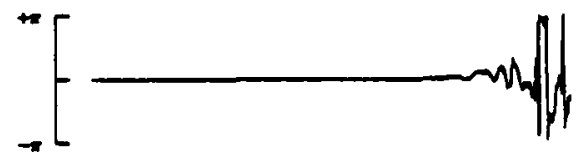

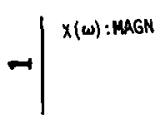

$\mathbf{N}$
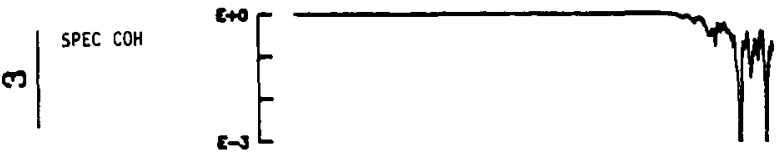

Fig. 6-Transfer function for base signal with the unfiltered base signal, with noise, so that it is similar to noise 1 , but reduced so that the signal-to-noise ratio is 2.5 percent instead of 30 percent. Figure includes transfer-function magnitude $[X(\omega)$ :MAGN], transfer-function angle $[X(\omega)$ : $A N G L)$, and spectral coherence for the signal with itself (SPEC COH) 


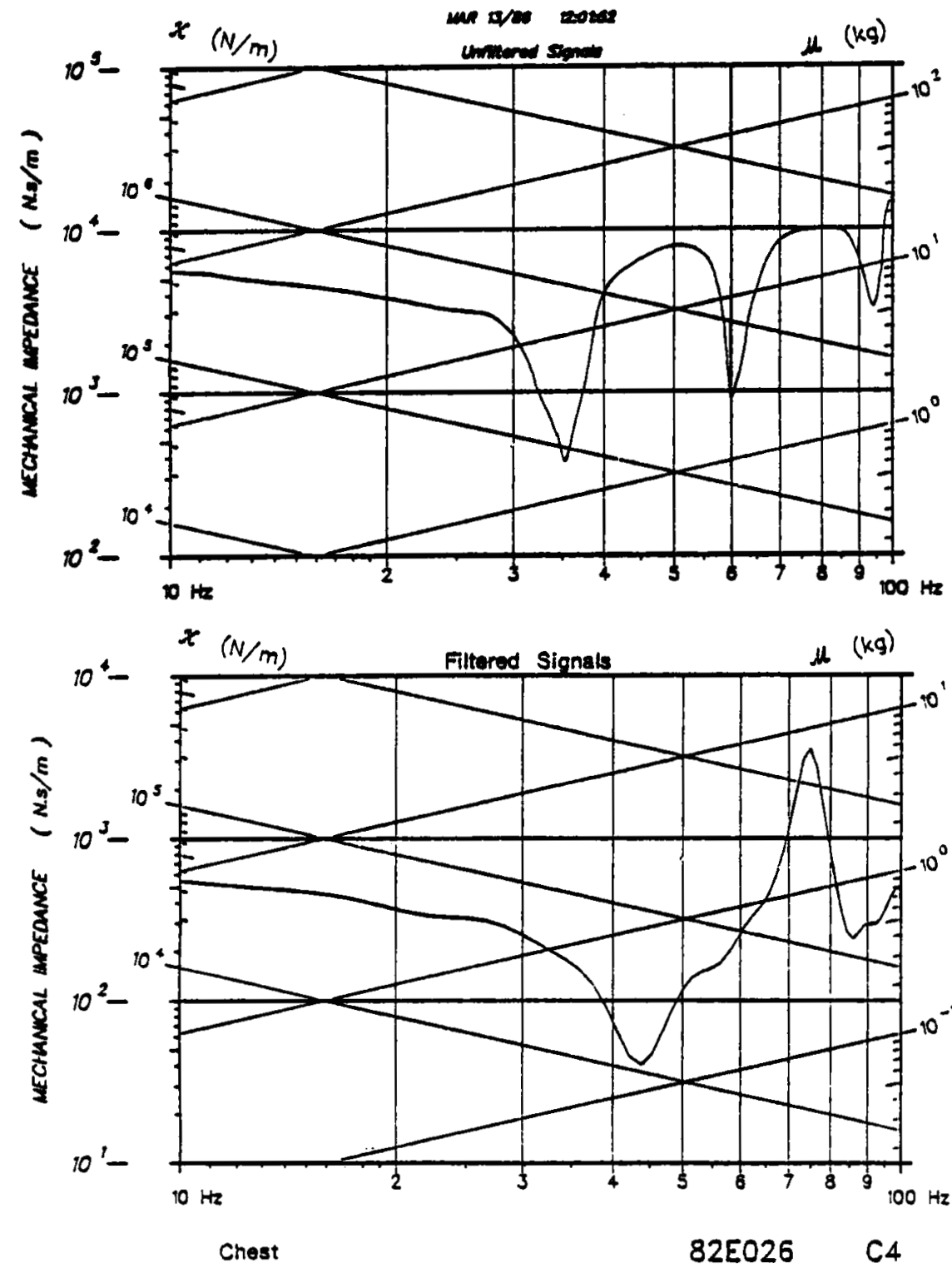

Fig. 7-Transfer function of an impact to the sternum of a repressurized cadaver. The transfer function is generated by using the impact force and the sternal accelerometer. The top figure is a transfer function that has been generated without using the filter. The bottom figure is a transfer function which is generated using the filter

time-histories with very successful results. It has shown itself to be conservative in that it never adds noise to a given signal. It does have one draw. back; the current algorithm is slow and no doubt could be speeded up. Means of speeding up the processing are currently being investigated.

\section{EXAMPLE}

The following example illustrates the use of the UMTRI filter. A signal that represents the contact-force time-history generated when a $25 \cdot \mathrm{kg}$ mass strikes the thorax of a repressurized cadaver was created. The signal was constructed from hyperbolic secants which were least-squares fitted to an actual thoracic time-history obtained from a blunt-im- pact experiment conducted at UMTRI. This signal was then used as the unaltered thoracic time-history and is referred to as the "base signal." Figure 1 illustrates a transfer function of the base signal with itself. Figure 2 illustrates the thoracic time-history of the base signal contrasted with the base signal having several different types of noise added as well as contrasted with those noisy signals run through the filter.

In the example presented here (Fig. 2), to obtain a reasonably well-defined transfer function, the noise introduced into the base signal cannot be eliminated to the degree necessary through the use of the filter, although there is significant improvement in the transfer function. Figure 3 is a transfer function of the base signal with the base signal with noise 2 from Fig. 2. (Note that the signal with the introduced noise always has more power at the higher frequencies than the base signal. This is an artifact of the way in which the noise was added.) Figure 4 is a transfer function of the filtered signal number 4 from Fig. 2 and the base signal.

In order to obtain a useful transfer function for this example, the original signal-to-noise ratio has to be approximately 50-to-1. This is illustrated in Figs. 5 and 6. For Figs. 5 and 6 , the signal that is used, in contrast to the base signal, has noise similar to that of signal number 5 in Fig. 2 (noise 1), but the introduced signal-to-noise level has been reduced from 3-to-1 to 50-to-1. Figure 5 contains the transfer function between the filtered signal and the base signal. Figure 6 contains the transfer function between the unfiltered signal and the base signal.

Figure 7 represents a transfer function for an impact to the sternum of a repressurized human cadaver. The top graph represents the unfiltered data and the bottom graph represents the filtered response. It can be seen that there is a significant change in the quality of the transfer function.

\section{ACKNOWLEDGMENTS}

This work was conducted under the sponsorship of the National Highway Traffic Safety Administration, Contract Nos. DTNH22-83-C-17019 and DTNH2283-C-07095. The author gratefully acknowledges the contributions of Robert Bennett, Richard Lehman, Valerie Karime, Bryan Suggitt, and Patricia Kaiker. Also, a special thanks is due to Gail Muscott who was responsible for implementing much of the code associated with the filter.

The protocol for the use of cadavers used in these experiments was approved by The University of Michigan Medical Center and followed guidelines established by the U.S. Public Health Service and those recommended by the National Academy of Sciences, National Research Council. Animals were handled according to the American Association for the Accreditation of Laboratory Animal Care and National Institutes of Health guidelines.

\section{REFERENGES}

1. Crede, C.E. and Harris, C.M., editors, "Shock and Vibration Handbook," 2nd edition, McGraw-Hill, Inc. (1976). 